\title{
ANALYSIS OF TWO-LEVEL DOMAIN DECOMPOSITION PRECONDITIONERS BASED ON AGGREGATION
}

\author{
MARZIO SALA ${ }^{1}$
}

\begin{abstract}
In this paper we present two-level overlapping domain decomposition preconditioners for the finite-element discretisation of elliptic problems in two and three dimensions. The computational domain is partitioned into overlapping subdomains, and a coarse space correction is added. We present an algebraic way to define the coarse space, based on the concept of aggregation. This employs a (smoothed) aggregation technique and does not require the introduction of a coarse grid. We consider a set of assumptions on the coarse basis functions, to ensure bound for the resulting preconditioned system. These assumptions only involve geometrical quantities associated to the aggregates, namely their diameter and the overlap. A condition number which depends on the product of the relative overlap among the subdomains and the relative overlap among the aggregates is proved. Numerical experiments on a model problem are reported to illustrate the performance of the proposed preconditioners.
\end{abstract}

Mathematics Subject Classification. 65M55, 65Y05.

Received: May 2, 2003. Revised: April 19, 2004.

\section{INTRODUCTION}

In this paper we consider the scalar Poisson problem

$$
\left\{\begin{aligned}
-\Delta u=f & \text { in } \Omega \\
u=0 & \text { on } \partial \Omega
\end{aligned}\right.
$$

where $\Omega$ is a bounded polyhedral domain in $\mathbb{R}^{d}, d \in\{2,3\}$. The finite-element discretisation of this problem leads to a sparse, large linear system, which is typically solved using an iterative solver, like the conjugate gradient. As the condition number of the associated matrix is $\mathcal{O}\left(h^{-2}\right)$ (see for instance [15]), where $h$ is the grid size of the triangulation, a preconditioner needs to be employed. A possible solution, well-suited for parallel computations, is to resort to preconditioners based on domain decomposition (DD) ideas, see [16,19].

Here, we consider Schwarz procedures, which can be described as follows. The computational domain $\Omega$ is subdivided into $M$ overlapping subdomains $\Omega_{i}, i=1, \ldots, M$, and local Dirichlet-type problems are then solved on each $\Omega_{i}$. The communication between the solutions on the different subdomains is here guaranteed by the overlapping regions.

Keywords and phrases. Elliptic equations, domain decomposition, Schwarz methods, aggregation coarse corrections.

1 CMCS/SB/EPFL, 1015 Lausanne, Switzerland. e-mail: msala@sandia.gov 
It is well-known that this procedure, referred to as one-level Schwarz method, is non-scalable. In fact, the information exchange among the subdomains is only through the overlapping regions, while for elliptic problems the domain of dependence is global. A good scalability may be recovered by the addition of a coarse operator: the original problem is solved on a coarse grid, to furnish a way to spread out information among far away subdomains. Thinking as in a multigrid setting, this coarse space will be used to correct the "smooth" part of the error, whereas the local preconditioner is used to damp the "high-frequency" ones.

A typical choice for the coarse problem is a finite-element approximation on a coarse grid. For structured grids, it is relatively easy to find such a coarse triangulation. For unstructured grids, it is not always a trivial task to define the interpolation operator from the fine grid to the coarse grid, and it may be problematic to ensure that the boundary conditions are correctly represented on the coarse level. Moreover, the implementation of this operator can be difficult or computationally expensive, especially for 3D computations.

A possible way to overcome the difficulties induced by the definition of a coarse grid - without losing the power of two-level methods - is to resort to aggregation procedures. As presented in this paper, aggregation (or agglomeration) is of particular advantage when dealing with problems defined on unstructured grids, since it is possible to introduce coarse spaces without the need of introducing coarse triangulations.

The procedure is as follows. First, the differential problem is discretized on the fine grid. Then, the matrix corresponding to the discretisation of the differential operator on the coarse space is build using the elements of the fine-grid matrix. The basis functions of the coarse space are formed by summing up the finite element basis functions of the nodes in each aggregate.

Aggregation procedures have been presented in literature by various authors. An aggregation technique was first introduced in 1951 by Leontief ([12], Chap. 9). Here, it is written that "in the case of products which comprise final demand, if enough is known to form a group of products whose use is strictly complementary [...], no information is sacrificed by lumping them together. By the same token, products which are substitutable but have dissimilar production functions must be separately identified since a change in their relative consumption will have different effects on the economy". Since then, aggregation has been extensively used in Economics; see [13] and the references therein. For multigrid applications, smoothed aggregation techniques are used, for example, in $[2,23]$, where investigations of the smoothed aggregation properties have been reported. In the framework of domain decomposition methods, the focus is mainly on two-level methods. Results are presented in [14] for the shallow water equations and 2D potential flows, in [6] for 3D potential flow computations, in [8] for groundwater flows, in [9] for multiphase flows, in [10] for discontinuous Galerkin approximation of advectiondiffusion problems, and in $[17,18]$ for the 3D compressible Euler equations on unstructured tetrahedral grids.

The basic theory and the assumptions on the smoothed aggregation procedures here presented are essentially the same as those already proposed in some recent articles; see [1,8,11]. However, in these articles the amount $\delta$ of the overlap among the subdomains is assumed to be equal to that among the aggregates, here indicated by $\delta_{0}$. Also, the size of the subdomains $H$ is assumed to be equal to that $H_{0}$ of the aggregates. In this paper, instead, we keep these two ingredients separate, thus extending the previously developed theory. This allows to better underline the different phases of our smoothed aggregation algorithm: the definition of a subdomain partition, and the definition of the aggregates. Effects of these two phases are reflected on the proposed bound of the condition number which involves geometrical quantities on the subdomains $(H$ and $\delta)$ and on the aggregates $\left(H_{0}\right.$ and $\left.\delta_{0}\right)$.

For the sake of completeness, for the special case of $H=H_{0}$ and $\delta=\delta_{0}$ we report an improved convergence bound, as proposed in [11]. In this case, the bound depends linearly on $H / h$, and this seems to be confirmed by our numerical results. However, it was not possible to extend the proof of [11] to our general setting.

The paper outline is as follows. Section 2 introduces the problem and the basic results of two-level Schwarz preconditioners. The definition of the coarse space using the concept of aggregation is considered in Section 3 . In Section 4, a theoretical estimate of the condition number for a model problem is given. Section 5 reports the improved convergence bound presented in [11]. Section 6 reports several numerical results for a model problem. Finally, conclusions are drawn in Section 7. 


\section{Model problem}

Let us consider again the Poisson problem (1), whose finite-element approximation reads

$$
\left\{\begin{array}{l}
\text { Find } u_{h} \in V_{h} \text { such that } \\
a\left(u_{h}, v_{h}\right)=f\left(v_{h}\right) \quad \forall v_{h} \in V_{h},
\end{array}\right.
$$

with $a\left(u_{h}, v_{h}\right)=\int_{\Omega} \nabla u_{h} \cdot \nabla v_{h} \mathrm{~d} \Omega, f\left(v_{h}\right)=\int_{\Omega} f v_{h} \mathrm{~d} \Omega$. By assumption, $f \in L_{2}(\Omega)$, and $V_{h}$ is a family of finite dimensional subspaces of $H_{0}^{1}(\Omega)$. Precisely, $V_{h}$ is the space of linear finite-element functions defined on a conforming, quasi-uniform triangulation $\mathcal{T}_{h}$ of $\Omega$. By notation, $\varphi_{i}$ is a generic basis function of $V_{h}$ and $h$ the grid size parameter. Existence of a unique solution of (2) is shown, for instance, in [15].

The computational domain $\Omega$ is then partitioned into $M$ overlapping subdomains $\Omega_{i}$. On each $\Omega_{i}$ we have, by construction, $\operatorname{diam}\left(\Omega_{i}\right) \leq C H$. Here and in the following, $C$ is a constant independent of $H$, $h$, the overlap among the subdomains $\delta$, and the coarse space parameters $H_{0}, \delta_{0}$, and $n_{0}$ defined below.

The algebraic formulation of problem (2) reads

$$
A \mathbf{u}=\mathbf{f}
$$

where $A \in \mathbb{R}^{n \times n}$ is a symmetric positive definite matrix, $\mathbf{u} \in \mathbb{R}^{n}$ the solution vector, and $\mathbf{f} \in \mathbb{R}^{n}$ the discretisation of force term $f$.

Let $V_{i} \subset H_{0}^{1}\left(\Omega_{i}\right)$ be a subspace of $V_{h}$ containing the finite element functions defined on each subdomain $\Omega_{i}$, $i=1, \ldots, M$. We can now define the matrices $B_{i}=R_{i}^{T} A_{i}^{-1} R_{i}$, where $A_{i}=R_{i} A R_{i}^{T}$ and $R_{i}^{T}$ is the discrete representation of the interpolation from $V_{i}$ to $V_{h}$. With this notation, the one-level additive Schwarz method can be regarded as preconditioned iterative method (ideally, the preconditioned conjugate gradient method if $A$ is symmetric and positive definite) for solving (3) with a preconditioner $P_{S}$ such that

$$
P_{S}^{-1}=\sum_{i=1}^{M} B_{i}
$$

In a two-level Schwarz method, a further term $B_{0}=R_{0}^{T} A_{0}^{-1} R_{0}$ is added to the preconditioner, obtaining

$$
P_{S, C, \text { add }}^{-1}=\sum_{i=0}^{M} B_{i}=\sum_{i=0}^{M} R_{i}^{T} A_{i}^{-1} R_{i}
$$

$A_{0}$ corresponds to the solution of the original variational problem in the space $V_{0}$, which is "coarse" in the sense that it contains a limited number of degrees of freedom, in order to make the "exact" inversion of $A_{0}$ computationally acceptable - if $n_{0}$ is the dimension of the coarse space, one must have $n_{0} \ll n$.

An alternative two-level method, derived from a three-step Richardson method, leads to the following preconditioned matrix:

$$
P_{C, \text { hybrid }}^{-1} A=I-\left(I-B_{0} A\right)\left(I-P_{S}^{-1} A\right)\left(I-B_{0} A\right) \text {. }
$$

This approach is very close to the so-called balancing Neumann-Neumann method for non-overlapping decompositions [20]. If the roles of $B_{0}$ and $P_{S}$ are interchanged, the resulting preconditioner is close to the multigrid approach, with $P_{S}$ playing the role of the smoother.

Remark 1. We always assume exact solvers for both the local and the coarse problems; approximate solvers could be considered as well with minor modifications. 
We assume that there exists a set of interpolation operators $\mathcal{I}_{i}: V_{i} \rightarrow V_{h}$, and that a continuous and coercive bilinear form $a_{i}(\cdot, \cdot)$ that approximates $a(\cdot, \cdot)$ is defined on each $V_{i}$. Finally, let the projection-like operators $\tilde{\mathcal{T}}_{i}: V_{h} \rightarrow V_{i}$ be defined as

$$
a_{i}\left(\tilde{\mathcal{T}}_{i} u, v\right)=a\left(u, \mathcal{I}_{i} v\right) \quad \forall v \in V_{i}, i=1, \ldots, M
$$

The properties of additive Schwarz preconditioner (5) can be studied using an abstract convergence theory, as presented in [5]; see also [19] and the references therein. This theory is based on the following 3 assumptions.

Assumption 1. Let $C_{0}$ be the minimum constant such that for all $u_{h} \in V_{h}$ there exists a representation $u_{h}=\sum_{i=0}^{M} \mathcal{I}_{i} u_{i}, u_{i} \in V_{i}$ such that

$$
\sum_{i=0}^{M} a_{i}\left(u_{i}, u_{i}\right) \leq C_{0}^{2} a\left(u_{h}, u_{h}\right) .
$$

Assumption 2. Define $0 \leq \epsilon_{i, j} \leq 1$ to be the minimal values that satisfy

$$
\left|a\left(\mathcal{I}_{i} u_{i}, \mathcal{I}_{j} u_{j}\right)\right| \leq \epsilon_{i, j} a\left(\mathcal{I}_{i} u_{i}, \mathcal{I}_{i} u_{i}\right)^{1 / 2} a\left(\mathcal{I}_{j} u_{j}, \mathcal{I}_{j} u_{j}\right)^{1 / 2} \quad \forall u_{i} \in V_{i}, \quad \forall u_{j} \in V_{j}, i, j=1, \ldots, M .
$$

We define $\rho(\epsilon)$ to be the spectral radius of a matrix $\epsilon$ whose entries are the $\epsilon_{i, j}$.

Assumption 3. Let $\omega$ be the minimum constant such that

$$
a\left(\mathcal{I}_{i} u_{i}, \mathcal{I}_{i} u_{i}\right) \leq \omega a_{i}\left(u_{i}, u_{i}\right) \quad \forall u_{i} \in V_{i}, \quad i=0, \ldots, M
$$

where we assume that the $a_{i}(\cdot, \cdot)$ are suitably scaled.

The following theorem holds. By notation, $\kappa(B)$ denotes the the spectral condition number of the square matrix $B$. For the proof, we refer to [5].

Lemma 1. The abstract additive Schwarz preconditioner satisfies

$$
\kappa\left(P_{S, C, \text { add }}^{-1} A\right) \leq \omega[1+\rho(\epsilon)] C_{0}^{2} .
$$

In particular, $1 / C_{0}^{2}$ is a sharp lower bound on the smallest eigenvalue of $P_{S, C \text {,add }}^{-1} A$ and $\omega[1+\rho(\epsilon)]$ is a bound on the largest eigenvalue of $P_{S, C, \text { add }}^{-1} A$.

\section{Definition OF THE COARSE SPACE}

The spectral properties (and the parallel performance) of the two-level Schwarz preconditioner will depend on the definition of the coarse space $V_{0}$. There are virtually unlimited choices of the coarse grid correction that may be used. Convergence of the entire scheme will depend on the particular interpolation and coarse grid operator used. When possible, this coarse space $V_{0}$ may be itself embedded into $V_{h}$. If a coarse grid is used to define $V_{0}$, the following result holds [19].

Theorem 1. For the additive two-level overlapping Schwarz method, when the overlap is uniform of width $\mathcal{O}(\delta)$, the coarse grid space $V_{0}$ corresponds to the finite-element functions on elements of $\mathcal{O}(H)$, and $V_{0} \subset V_{h}$, we have:

$$
\kappa\left(P_{S, C, \text { add }}^{-1} A\right) \leq C\left(1+\frac{H}{\delta}\right),
$$

where $C$ is a constant independent of $h, H$ and $\delta$. 

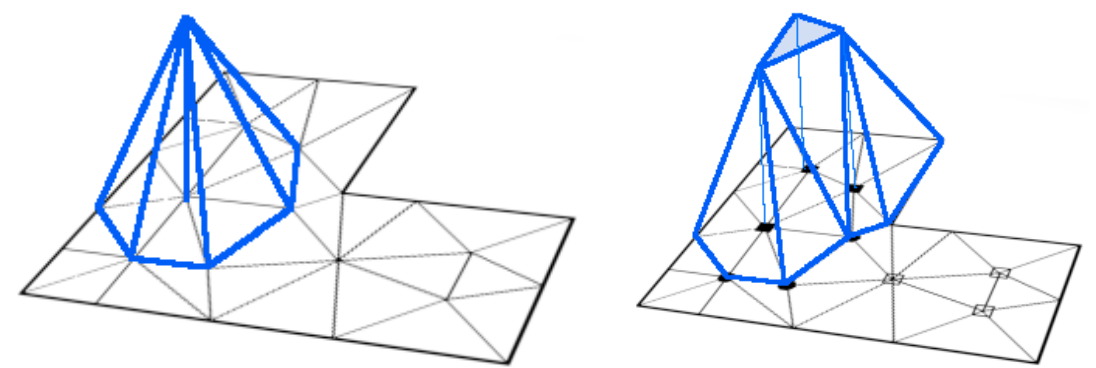

Figure 1. Example of basis function for the fine grid (on the left), and non-smoothed basis function for the coarse space (on the right).

In aggregation procedures, instead, the definition of the coarse space does not require the explicit construction of a coarse triangulation, since each element of $V_{0}$ is actually composed by a linear combination of elements of $V_{h}$. More precisely, we will group the nodes of the fine grid into $n_{0}$ sets of contiguous nodes, called aggregates. Then, the basis functions of the coarse space $V_{0}$ will be formed by summing up the finite element basis functions of all the nodes belonging to each aggregate. This defines the restriction operator $R_{0}$. The coarse matrix is computed as $A_{0}=R_{0} A R_{0}^{T}$.

To define $R_{0}$, we start by constructing a tentative, non-smoothed restriction $\tilde{R}_{0}$, so that each fine grid node is included in just one aggregate. This decomposition can be obtained using a graph partitioning algorithm (using for instance a vertex-oriented decomposition of the grid). The number of aggregates $n_{0}$ will represent the dimension of the coarse space $V_{0}$, since each aggregate will be given a single coarse grid basis function. We will indicate with $\tilde{\vartheta}_{i}$ the set of nodes that form the non-smoothed aggregate $i$. The entries of $\tilde{R}_{0}$ are thus as follows:

$$
\tilde{R}_{0}(i, j)= \begin{cases}1 & \text { if } j \in \tilde{\vartheta}_{i} \\ 0 & \text { otherwise }\end{cases}
$$

$\tilde{R}_{0}$ can be viewed as a simple grid transfer operator corresponding to piecewise constant interpolation. With this technique $V_{0} \subset V_{h}$ and the non-smoothed basis functions $\left\{\tilde{\Phi}_{i}\right\}$ of $V_{0}$ are defined as

$$
\tilde{\Phi}_{i}(\mathbf{x})=\sum_{j \in \tilde{\vartheta}_{i}} \varphi_{j}(\mathbf{x})
$$

A 2D example is reported in Figure 1.

Once $\tilde{R}_{0}$ has been defined, the second step (that may actually be avoided) consists of applying a prolongator smoother $\tilde{S}_{0}$ to produce the final prolongator operator $R_{0}^{T}=\tilde{R}_{0}^{T} \tilde{S}_{0}$. The idea is to smooth out the $\left\{\tilde{\Phi}_{i}\right\}$, to reduce their $H_{1}$-norm (this also results in an increased support), using the stencil of suitable polynomials in $A$. The final coarse space reads

$$
V_{0}:=\operatorname{span}\left\{\Phi_{i}\right\}=\operatorname{span}\left\{\tilde{S}_{0} \tilde{\Phi}_{i}\right\} .
$$

A possible choice for the smoother is the Richardson smoother $[10,11]$ (where $A$ may need to be suitably scaled):

$$
\tilde{S}=\tilde{S}_{0}\left(\varpi_{k}, k\right)=\left(I-\varpi_{k} A\right)^{k},
$$

where $\varpi_{k} \in(0,1]$ is a real parameter and $k \in \mathbb{N}_{0}$. Unless $A$ has particular properties (for example, it is a $M$-matrix), the spectral radius of a non-damped Richardson method is greater than one, hence the smoother requires the definition of a proper value for $\varpi$. In this case, $\varpi$ must be chosen such that $\varpi<2 / \rho(A)$. Usually, it is not too difficult to obtain a good estimate of $\rho(A)$, using for instance the Gershgorin theorem, a small number 
of Lanczos or conjugate gradient or power method iterations. It is sometimes useful to scale the estimated value by a small factor, because most computational methods give lower bounds to the largest eigenvalue.

Other smoothers proposed in literature are a recursive Richardson smoother [2,22], or a SPAI smoother [3]. For a general comparison of these smoothers the reader is addressed to [10] or [11], where it is shown that, for the considered model problem, the effect of the above mentioned smoothers is quite similar.

\section{A CONVERGENCE BOUnd}

In order to provide a bound for the two-level Schwarz preconditioner with aggregation coarse space, we need to make precise some geometrical information about the used decomposition. This is done in the following properties. By notation, we indicate with $\vartheta_{j}$ the set of nodes belonging to the $j$-smoothed aggregate, with $\Theta_{j}=\operatorname{int}\left(\cup_{i \in \vartheta_{j}} \operatorname{supp}\left(\varphi_{i}\right)\right)$ the support of $\Phi_{j}, j=1, \ldots, n_{0}$, and with $H_{0}=\max _{j}\left\{\operatorname{diam}\left(\Theta_{j}\right)\right\}$. In the following we will use the term aggregate for both $\vartheta_{j}$ and $\Theta_{j}$, depending on the context.

Property 1 (partition). There exist two constants $C_{1}$ and $C_{2}$ so that, for each aggregate $\Theta_{i}, i=1, \ldots, n_{0}$, we have:

a. $\operatorname{diam}\left(\Theta_{i}\right) \leq C_{1} H_{0}$

b. the Lebesgue measure $\left|\Theta_{i}\right|$ of $\Theta_{i}$ satisfies $\left|\Theta_{i}\right| \geq C_{2} H_{0}^{d}$;

c. the overlap among the aggregates is of order $\delta_{0}$.

Property 1 states that the aggregates have diameter of comparable size $H_{0}$ and are shape-regular. The following Property 2 requires a certain regularity on the coarse space basis function $\Phi_{i}$.

Property 2 (coarse space). We assume that the basis functions $\left\{\Phi_{i}\right\}$ of the coarse space satisfy

a. $\left|\Phi_{i}\right|_{H^{1}(\Omega)}^{2} \leq C \frac{H_{0}^{d-1}}{\delta_{0}} ;$

b. $\left\|\Phi_{i}\right\|_{L^{2}(\Omega)}^{2} \leq C H_{0}^{d}$;

c. there is a domain $\hat{\Omega} \subset \Omega$ such that $\sum_{i} \Phi_{i}(x)=1$ for every $x \in \hat{\Omega}$ and $\sup _{x \in \Omega \backslash \hat{\Omega}} \operatorname{dist}(x, \partial \Omega) \leq C H_{0}$.

We can now prove the following lemma.

Lemma 2. Let us define the operator $Q_{0}: V_{h} \rightarrow V_{0}$ by

$$
Q_{0} u=\sum_{i=1}^{n_{0}} \alpha_{i} \Phi_{i}, \quad \alpha_{i}=\frac{1}{\left|\Theta_{i}\right|} \int_{\Theta_{i}} u(\mathbf{x}) \mathrm{d} \Omega .
$$

If Properties 1 and 2 are satisfied, then there exists a constant $C>0$ independent of $H_{0}, \delta_{0}$ and $h$ such that $\forall u \in V_{h}$

a. $\left\|u-Q_{0} u\right\|_{L^{2}(\Omega)}^{2} \leq C H_{0}^{2}|u|_{H^{1}(\Omega)}^{2} ;$

b. $\left|Q_{0} u\right|_{H^{1}(\Omega)}^{2} \leq C \frac{H_{0}}{\delta_{0}}|u|_{H^{1}(\Omega)}^{2}$.

Proof. The proof extends a similar proof proposed in [8]. It is split into 2 parts. First, we prove Lemma 2 in $\Omega \backslash \hat{\Omega}$; then, in $\hat{\Omega}$, where $\hat{\Omega}$ is the domain introduced in Property 2. Throughout the proof, $C$ represents a generic positive constant independent of $h, H, H_{0}, \delta$, and $\delta_{0}$.

First, we note that, for the operator $Q_{0}$ defined by (15), we have

$$
\sum_{i=1}^{n_{0}} \alpha_{i}^{2}(u) \leq C H_{0}^{-d}\|u\|_{L^{2}(\Omega)}^{2}
$$

which easily follows by the Cauchy-Schwarz inequality. 

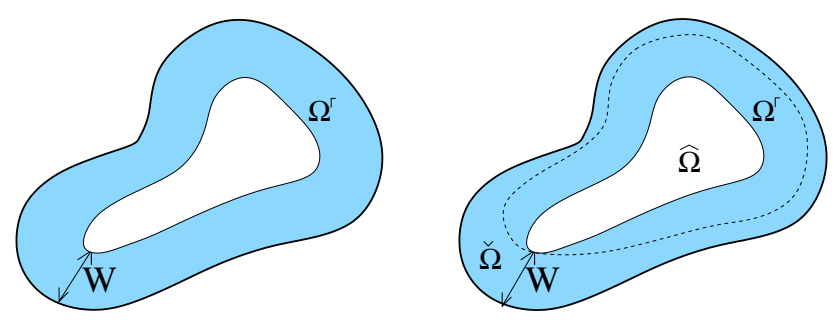

Figure 2. On the left, in shaded colour, $\Omega^{\Gamma}$. On the right, in shaded colour, $\check{\Omega}$, and in white, $\hat{\Omega}$.

Now, let us consider Lemma $2 \mathrm{a}$, and set $\check{\Omega}=\Omega \backslash \hat{\Omega}$. Further, define

$$
\mathcal{B}=\left\{i: \Theta_{i} \cap \check{\Omega} \neq \emptyset\right\}, \quad \check{\Xi}=\bigcup_{i \in \mathcal{B}} \Theta_{i}, \quad W=\sup _{x \in \check{\Omega}} \inf _{y \in \partial \Omega} \operatorname{dist}(x, y)
$$

and set $\Omega^{\Gamma}=\{x \in \Omega \mid \operatorname{dist}(x, \partial \Omega) \leq W\}$. Figure 2 illustrates the domains $\check{\Omega}$ and $\Omega^{\Gamma}$.

From Property 1c it follows that $W \leq \mathrm{CH}_{0}$, and therefore the Poincaré inequality yields

$$
\|u\|_{L_{2}(\check{\Omega})} \leq\|u\|_{L^{2}\left(\Omega^{\Gamma}\right)} \leq C H_{0}|u|_{H^{1}\left(\Omega^{\Gamma}\right)} .
$$

The restriction of $Q_{0} u$ onto $\check{\Omega}$ can be expressed as

$$
\left.\left(Q_{0} u\right)\right|_{\check{\Omega}}=\left.\sum_{i \in \mathcal{B}} \alpha_{i}(u) \Phi_{i}(x)\right|_{\check{\Omega}}
$$

Further, let us set

We have

$$
\mathcal{N}_{i}=\left\{j \mid \Theta_{j} \cap \Theta_{i} \neq \emptyset\right\}
$$

[by Prop. 2b]

$$
\begin{aligned}
\left\|Q_{0} u\right\|_{L^{2}(\check{\Omega})}^{2} & =\sum_{i \in \mathcal{B}} \sum_{j \in \mathcal{N}_{i} \cap \mathcal{B}}\left(\alpha_{i}(u) \Phi_{i}, \alpha_{j}(u) \Phi_{j}\right)_{L^{2}(\check{\Omega})} \\
& \leq \sum_{i \in \mathcal{B}} \sum_{j \in \mathcal{N}_{i} \cap \mathcal{B}}\left|\alpha_{i}(u)\right| \cdot\left|\alpha_{j}(u)\right| \cdot\left\|\Phi_{i}\right\|_{L^{2}(\Omega)}\left\|\Phi_{j}\right\|_{L^{2}(\Omega)} \\
& \leq C H_{0}^{d} \sum_{i \in \mathcal{B}} \sum_{j \in \mathcal{N}_{i} \cap \mathcal{B}} \frac{1}{2}\left(\alpha_{i}^{2}(u)+\alpha_{j}^{2}(u)\right) \\
& \leq C H_{0}^{d} \max \left\{\operatorname{card} \mathcal{N}_{i}\right\} \sum_{i \in \mathcal{B}} \alpha_{i}^{2}(u) \\
& \leq C\|u\|_{L^{2}\left(\Omega^{\Gamma}\right)}^{2} .
\end{aligned}
$$

[by (16)]

Using the last inequality together with inequality (17) gives

$$
\left\|u-Q_{0} u\right\|_{L^{2}(\check{\Omega})} \leq C\|u\|_{L^{2}(\check{\Omega})} \leq C H_{0}|u|_{H^{1}\left(\Omega^{\Gamma}\right)} .
$$

In an analogous way, we can estimate $2 \mathrm{~b}$ in $\check{\Omega}$ exploiting Property $2 \mathrm{a}$, obtaining

$$
\left|Q_{0} u\right|_{H^{1}(\check{\Omega})}^{2} \leq C H_{0}^{-1} / \delta_{0}\|u\|_{L^{2}\left(\Omega^{\Gamma}\right)}^{2} \leq C H_{0} / \delta_{0}|u|_{H^{1}\left(\Omega^{\Gamma}\right)}^{2} .
$$

Now, we turn our attention to $\hat{\Omega}$. 
For every function $u \in V_{h}$, let us consider an extension $u_{E}$ satisfying

$$
\left\|u_{E}\right\|_{H^{1}\left(\mathbb{R}^{d}\right)} \leq\|u\|_{H^{1}(\Omega)}, \quad u_{E}=u \text { in } \Omega .
$$

For $j=1, \ldots, n_{0}$, we define $\Theta_{j}^{\star}=\cup_{i \in \mathcal{N}_{j}} \Theta_{i}$ with $\mathcal{N}_{i}$ given by (18), and $\Xi_{j}$ to be the ball circumscribing $\Theta_{j}^{\star}$. From property 1 is follows that $\operatorname{diam}\left(\Xi_{j}\right) \leq C H_{0}$. We now use the Friedrichs inequality in the form

$$
\|u\|_{L^{2}\left(\Xi_{j}\right)} \leq C H_{0}|u|_{H^{1}\left(\Xi_{j}\right)}, \quad \forall u \in\left\{v \in H^{1}\left(\Xi_{j}\right) \mid \int_{\Xi_{j}} v \mathrm{~d} x=0\right\} .
$$

For every $j=1, \ldots, n_{0}$ we define

$$
c_{j}=\int_{\Xi_{j}} u_{E} \mathrm{~d} x, \quad \bar{u}_{j}=u_{E}-c_{j} .
$$

Then, the Friedrichs inequality holds for every $\bar{u}_{j}$. Due to Property 2 c, for every $x \in \Theta_{j} \cap \hat{\Omega}$ it holds

[by Prop. 2c]

$$
\begin{aligned}
\left(Q_{0} u\right)(x) & =\left(Q_{0} \bar{u}_{j}\right)(x)+Q_{0} c_{j} \\
& =\sum_{i \in \mathcal{N}_{j}} \alpha_{i}\left(\bar{u}_{j}\right) \Phi_{i}(x)+c_{j} \sum_{i \in \mathcal{N}_{j}} \Phi_{i}(x) \\
& =\left(Q_{0} \bar{u}_{j}\right)(x)+c_{j} .
\end{aligned}
$$

Therefore,

$$
\begin{aligned}
\left\|u-Q_{0} u\right\|_{L^{2}(\hat{\Omega})}^{2} & \leq=\sum_{i}\left\|\left(I-Q_{0}\right)\left(\bar{u}_{i}+c_{i}\right)\right\|_{L^{2}\left(\Xi_{i} \cap \hat{\Omega}\right)}^{2} \\
& \leq 2 \sum_{i}\left(\left\|\bar{u}_{i}\right\|_{L^{2}\left(\Xi_{i} \cap \hat{\Omega}\right)}^{2}+\left\|Q_{0} \bar{u}_{i}\right\|_{L^{2}\left(\Xi_{i} \cap \hat{\Omega}\right)}^{2}\right) .
\end{aligned}
$$

Further,

[by $2 \mathrm{~b}$ and (16)]

$$
\begin{aligned}
\left\|Q_{0} \bar{u}_{i}\right\|_{L^{2}\left(\Xi_{i} \cap \hat{\Omega}\right)}^{2} & \leq\left\|\sum_{j \in \mathcal{N}_{i}} \alpha_{j}\left(\bar{u}_{i}\right) \Phi_{j}\right\|_{L^{2}(\Omega)}^{2} \\
& \leq\left(\sum_{j \in \mathcal{N}_{i}}\left|\alpha_{j}\left(\bar{u}_{i}\right)\right| \cdot\left\|\Phi_{j}\right\|_{L^{2}(\Omega)}\right)^{2} \\
& \leq \operatorname{card}\left\{\mathcal{N}_{j}\right\} \sum_{j \in \mathcal{N}_{i}} \alpha_{j}^{2}\left(\bar{u}_{i}\right)\left\|\Phi_{j}\right\|_{L^{2}\left(\Xi_{i} \cap \check{\Omega}\right)}^{2} \\
& \leq C\left\|\bar{u}_{i}\right\|_{L^{2}\left(\Xi_{i} \cap \check{\Omega}\right)}^{2} .
\end{aligned}
$$

Substituting the last inequality into (21), using the Friedrichs inequality (20) and exploiting the bounded intersections of balls $\left\{\Xi_{i}\right\}_{i=1}^{n_{0}}$, we get

$$
\left\|u-Q_{0} u\right\|_{L^{2}(\hat{\Omega})}^{2} \leq C \sum_{i=1}^{n_{0}}\left\|\bar{u}_{i}\right\|_{L^{2}\left(\Xi_{i}\right)}^{2} \leq C H_{0}^{2}|u|_{H^{1}(\Omega)}^{2} .
$$


We have therefore proved Lemma 2a, since

$$
\begin{aligned}
\left\|u-Q_{0} u\right\|_{L^{2}(\Omega)}^{2} & =\left\|u-Q_{0} u\right\|_{L^{2}(\check{\Omega})}^{2}+\left\|u-Q_{0} u\right\|_{L^{2}(\hat{\Omega})}^{2} \\
& \leq C H_{0}^{2}|u|_{H^{1}\left(\Omega^{\Gamma}\right)}^{2}+C H_{0}^{2}|u|_{H^{1}(\Omega)}^{2} \leq C H_{0}^{2}|u|_{H^{1}(\Omega)}^{2} .
\end{aligned}
$$

To finish the proof, we turn to $2 \mathrm{~b}$ in $\hat{\Omega}$. We have

[by Prop. 2a and (16)]

$$
\begin{aligned}
\left|Q_{0} u\right|_{H^{1}(\hat{\Omega})}^{2} & \leq \sum_{i=1}^{n_{0}}\left|Q_{0} u\right|_{H^{1}\left(\Theta_{i} \cap \hat{\Omega}\right)}^{2} \\
& \leq C \frac{H_{0}^{d-1}}{\delta_{0}} H_{0}^{-d} \sum_{i=1}^{n_{0}} \sum_{j \in \mathcal{N}_{i}}\left\|\bar{u}_{i}\right\|_{L^{2}\left(\Theta_{i}\right)}^{2} \\
& \leq C \frac{H_{0}}{\delta_{0}} \sum_{i=1}^{n_{0}}\left|\bar{u}_{i}\right|_{H^{1}\left(\Xi_{i}\right)}^{2} \\
& \leq C \frac{H_{0}}{\delta_{0}}|u|_{H^{1}(\Omega)}^{2} .
\end{aligned}
$$

For our main result we need the following two lemmas.

Lemma 3. Let $\Omega_{i} \subset \mathbb{R}^{d}, d=2,3$, be a rectangle of diameter $H$, and let $\Gamma_{\delta_{i}}$ be a strip along its boundary of width $\delta>0$. Then, for any function $u \in H^{1}\left(\Omega_{i}\right)$,

$$
\|u\|_{L^{2}\left(\Gamma_{\delta_{i}}\right)}^{2} \leq C \delta^{2}\left[\left(1+\frac{H}{\delta}\right)|u|_{H^{1}\left(\Omega_{i}\right)}^{2}+\frac{1}{H \delta}\|u\|_{L^{2}\left(\Omega_{i}\right)}^{2}\right] .
$$

Proof. See [4].

Lemma 4. Under Properties 1 and 2 and for every finite element function $u_{h} \in V_{h}$, there exists a decomposition $\left\{u_{i} \in V_{i}\right\}, i=0, \ldots, M$, such that

$$
u_{h}=\sum_{i=0}^{M} u_{i}
$$

and

with $H_{0} \leq H$ and $\delta_{0} \geq \delta$.

$$
\sum_{i=0}^{M}\left|u_{i}\right|_{H^{1}(\Omega)}^{2} \leq C\left(1+\frac{H}{\delta}\right)\left(1+\frac{H_{0}}{\delta_{0}}\right)\left|u_{h}\right|_{H^{1}(\Omega)}^{2},
$$

Proof. Define $I_{h}$ to be the fine grid operator $I_{h}: V \rightarrow V_{h}$ such that

$$
I_{h}\left(u_{h}\right)=\sum_{i=1}^{n} u_{h}\left(\mathbf{x}_{i}\right) \varphi_{i}
$$

where $\varphi_{i}$ is a generic finite element basis on the fine grid, and $\mathbf{x}_{i}$ 's are the fine grid nodal points. Let $\eta_{i}$ be a partition of unity such that $\eta_{i} \in C_{0}^{\infty}\left(\Omega_{i}\right)$ and $0 \leq \eta_{i} \leq 1$. We then define

$$
u_{0}=Q_{0} u_{h}, \quad w=u_{h}-u_{0}, \quad u_{i}=I_{h}\left(\eta_{i} w\right) .
$$

Equation (23) is verified by construction. Because of the definitions on the overlap, we can ensure that the gradients of $\eta_{i}$ are well behaved. That is, we can construct $\eta_{i}$ so that $\left|\nabla \eta_{i}\right|_{L^{\infty}(\Omega)}^{2} \leq C / \delta^{2}$. 
Over a single element $K$ and using the inverse inequality, we easily get

$$
\begin{aligned}
\left|u_{i}\right|_{H^{1}(K)}^{2} & =\left|I_{h}\left[\bar{\eta}_{i} w+\left(\eta_{i}-\bar{\eta}_{i}\right) w\right]\right|_{H^{1}(K)}^{2} \\
& \leq 2\left|\bar{\eta}_{i} w\right|_{H^{1}(K)}^{2}+2\left|I_{h}\left(\eta_{i}-\bar{\eta}_{i}\right) w\right|_{H^{1}(K)}^{2} \\
& \leq 2\left|\bar{\eta}_{i} w\right|_{H^{1}(K)}^{2}+C h^{-2}\left\|I_{h}\left(\eta_{i}-\bar{\eta}_{i}\right) w\right\|_{L^{2}(K)}^{2} \\
& \leq 2\left|\bar{\eta}_{i} w\right|_{H^{1}(K)}^{2}+C h^{-2}\left\|\eta_{i}-\bar{\eta}_{i}\right\|_{L^{\infty}(K)}^{2}\left\|I_{h} w\right\|_{L^{2}(K)}^{2}
\end{aligned}
$$

where $\bar{\eta}_{i}$ being the average of $\eta_{i}$ on element $K$. Now, we sum up over all the elements. The last term is identically zero for all elements $K$ in the interior of $\Omega_{i}$. Therefore, when we take the sum over all the elements, the last term only includes those elements in the overlapping region. Moreover, since a finite number, bounded independently of $h, \delta, H$ and $H_{0}$, of $u_{i}$ is non-zero for any element $K$, we obtain summing over $i$,

$$
\sum_{i=1}^{M}\left|u_{i}\right|_{H^{1}(\Omega)}^{2} \leq C|w|_{H^{1}(\Omega)}^{2} C \delta^{-2} \sum_{i=1}^{M}\left\|u_{i}\right\|_{L^{2}\left(\Gamma_{\delta_{i}}\right)}^{2} .
$$

Using Lemma 3 to bound the last term and Lemma 2, we get

$$
\begin{aligned}
\sum_{i=1}^{M}\left|u_{i}\right|_{H^{1}(\Omega)}^{2} & \leq C|w|_{H^{1}(\Omega)}^{2}+C \sum_{i=1}^{M}\left[\left(1+\frac{H}{\delta}\right)\left|u_{i}\right|_{H^{1}(\Omega)}^{2}+\frac{1}{H \delta}\left\|u_{i}\right\|_{L^{2}(\Omega)}^{2}\right] \\
& \leq C|w|_{H^{1}(\Omega)}^{2}+C\left(1+\frac{H}{\delta}\right)|w|_{H^{1}(\Omega)}^{2}+C \frac{1}{H \delta}\|w\|_{L^{2}(\Omega)}^{2} \\
& \leq C\left(1+\frac{H}{\delta}\right)\left|u-Q_{0} u\right|_{H^{1}(\Omega)}^{2}+C \frac{H_{0}^{2}}{H \delta}|u|_{H^{1}(\Omega)}^{2} \\
& \leq C\left(1+\frac{H}{\delta}\right)\left(1+\frac{H_{0}}{\delta_{0}}\right)|u|_{H^{1}(\Omega)}^{2} .
\end{aligned}
$$

Note that we have used the fact that $\delta_{0} \geq \delta$ and $H_{0} \leq H$. This latter inequality states that the coarse space must be sufficiently rich with respect to the number of subdomains.

The following theorem states the main result of this paper.

Theorem 2 (aggregation coarse space). Let the Properties 1 and 2 hold. Then, for the additive two-level overlapping Schwarz method, when the overlap is uniform of width $\mathcal{O}(\delta)$ and $V_{0}=\operatorname{span}\left\{\Phi_{i}, i=1, \ldots, n_{0}\right\}$, there exists $C>0$ such that

$$
\kappa\left(P_{S, C, \text { add }}^{-1} A\right) \leq C\left(1+\frac{H_{0}}{\delta_{0}}\right)\left(1+\frac{H}{\delta}\right) .
$$

Proof. The proof follows easily using Lemma 1 . As verified by Lemma 4, the first parameter $C_{0}^{2}$ of Definition 1 is bounded by

$$
C_{0}^{2} \leq\left(1+\frac{H}{\delta}\right)\left(1+\frac{H_{0}}{\delta_{0}}\right)
$$

The second parameter can be estimated by using a coloring argument. We know that we can colour the subdomains with $N_{c}$ colours, independently of $h$ and $H$. Taking the coarse space into account we have therefore that we can group the $T_{i}$ into $N_{c}+1$ classes. Hence, $\rho(\epsilon) \leq N_{c}+1$. Since $\omega=1$ as we suppose to use exact solvers on the subdomains, the thesis follows from Lemma 1.

Next, we report two lemmas, which prove that both the non-smoothed and the smoothed aggregation procedure outlined in Section 3 satisfy Properties 1 and 2. 
Lemma 5 (non-smoothed aggregation). The non-smoothed functions $\left\{\tilde{\Phi}_{i}\right\}$ defined by equation (13) satisfy Property 2 with $H_{0}=\tilde{H}_{0}$ and $\delta_{0}=h$.

Proof. Property 2a is proved by noting that $\nabla \tilde{\Phi}_{i}$ is zero outside the overlapping part $\Gamma_{\tilde{\Theta}_{i}}$ of $\tilde{\Theta}_{i}$ of width $h$, while it is bounded by $1 / h$ on $\Gamma_{\tilde{\Theta}_{i}}$, and that $\Gamma_{\tilde{\Theta}_{i}}$, where the gradient of $\tilde{\Phi}_{i}$ is non-zero, has a Lebesgue measure of order $\mathcal{O}\left(h \tilde{H}_{0}^{d-1}\right)$ and the grid is quasi-uniform. Property $2 \mathrm{~b}$ is shown noting that $\left\|\tilde{\Phi}_{i}\right\|_{L^{2}(\Omega)}^{2} \leq C\left|\tilde{\Theta}_{i}\right| \leq C \tilde{H}_{0}^{d}$. where $\tilde{\Theta}_{i}$ is the support of $\tilde{\Phi}_{i}$. Property 2c easily follows.

Lemma 6 (smoothed aggregation). Let $\tilde{S}_{0}$ be defined as in equation (14), and let $\tilde{\Phi}_{i}, i=1, \ldots, n_{0}$ satisfy Properties 1 and 2 with $H_{0}$ replaced by $\tilde{H}_{0}$ and $\delta_{0}$ by $\tilde{\delta}_{0}$. Then, for a fixed, sufficiently small, degree $k$ of the smoother, the coarse functions $\Phi_{i}=\tilde{S}_{0}(k) \tilde{\Phi}_{i}$ satisfy Properties 1 and 2.

Proof. The effect of the smoother is to increase the diameter of the subdomains and the overlap. The application of the smoother results in a diameter of the aggregates of $\mathcal{O}\left(\tilde{H}_{0}+k h\right)$ and an overlap of order $\mathcal{O}(h+k h)$. Since the triangulation is quasi-uniform, we have that, for a fixed $k$,

$$
\operatorname{diam}\left(\Theta_{i}\right)=C\left(\tilde{H}_{0}+k h\right) \leq C H_{0}
$$

This verifies Property 1a. Using similar arguments, Property $1 \mathrm{~b}$ is verified.

Now, let us prove that Property 2a holds. We have

$$
\left|\Phi_{i}\right|_{H^{1}(\Omega)}^{2}=\left|\tilde{S}_{0} \tilde{\Phi}_{i}\right|_{H^{1}(\Omega)}^{2} \leq \rho\left(\tilde{S}_{0}\right)^{2}\left|\tilde{\Phi}_{i}\right|_{H^{1}(\Omega)}^{2} \leq C \rho\left(\tilde{S}_{0}\right)^{2} \frac{H_{0}^{d-1}}{\delta_{0}}
$$

since $\tilde{H}_{0} \leq H_{0}$ and $\delta_{0}=h+k h \geq C \tilde{\delta}_{0}$ for a fixed $k$.

As regards Property $2 \mathrm{~b}$, we have:

$$
\left\|\Phi_{i}\right\|_{L^{2}(\Omega)}^{2}=\left\|\tilde{S}_{0} \tilde{\Phi}_{i}\right\|_{L^{2}(\Omega)}^{2} \leq \rho\left(\tilde{S}_{0}\right)^{2}\left\|\tilde{\Phi}_{i}\right\|_{L^{2}(\Omega)}^{2} \leq C H_{0}^{d} .
$$

To prove Property 2c, we define the function $u(x)=\sum_{i=1}^{n_{0}} \tilde{\Phi}_{i}(x)$, which is equal to one $\forall \mathbf{x} \in \hat{\Omega}$, and consequently at every $x \in \Omega$ outside a strip of width $\mathcal{O}\left(\tilde{\delta}_{0}\right)$ around $\partial \Omega$. Thus, we obtain

$$
\sum_{i=1}^{n_{0}} \Phi_{i}(x)=\left(\tilde{S}_{0} \sum_{i=1}^{n_{0}} \tilde{\Phi}_{i}\right)(x)=\left(\tilde{S}_{0} u\right)(x)=1
$$

at every $x \in \Omega$ outside a strip of width $\mathcal{O}(h)+k h=\mathcal{O}\left(\delta_{0}\right)$ around $\partial \Omega$.

We conclude this section with two remarks about the parallel implementation of SA techniques.

Remark 2. In general, one let each processor build the aggregate corresponding to its piece of the grid (corresponding to the triangulation of the computational domain), ignoring the connections among subdomains. That is, each processor is assigned a subgrid of the entire grid (that corresponds to the triangulation of the subdomain given to that processor); then, a serial aggregation algorithm is used on each subgrid. This approach, sometimes referred to as decoupled aggregation, is efficient if the load balance of the grid data among the processors is satisfactory. In general, when the ratio between the number of nodes and the number of aggregates is large enough, like for the two-level methods here presented, the decoupled aggregation offers good partitioning. If a large number of aggregates are required (like, for instance, in multilevel methods), decoupled aggregation may result in a somewhat irregular decomposition, and in this case it is usually worth to re-equilibrate the partitioning among the subdomains to minimize the dependency of the resulting algorithm on the subdomain decomposition; see [21]. 
Remark 3. If $\delta_{0}=\delta=\eta h, \eta \geq 1$, and the diameter of the aggregates $\Theta_{i}$ is bounded by $H / \chi$, where $\chi \geq 1$, that is, $\chi H_{0}=H$, we have

$$
\kappa\left(P_{S, C, \text { add }}^{-1} A\right) \leq C\left(1+\frac{H}{\eta h}\right)\left(1+\frac{H}{\chi \eta h}\right) \leq \frac{C}{\chi \eta^{2}}\left(\frac{H}{h}\right)^{2} .
$$

Equation (25) states that we can reduce the condition number by increasing the number of aggregates and the overlap. Computationally, it is expensive to use wider overlap (a minimal-overlap is often preferred), whereas larger values of $\chi$ have influence only on the coarse problem, whose size is remarkably smaller than that of the global problem.

\section{An improved CONVERGENCE BOUnd}

A possible improvement of the estimate given by Theorem 2 can be obtained considering that each subdomain defines an aggregate. This bound is presented in [11]. To establish this result, the following property is required.

Property 3. We assume that the basis functions $\left\{\Phi_{i}\right\}, i=0, \ldots, M$ of the coarse space $V_{0}$ satisfy

(1) $\left\|\Phi_{i}\right\|_{L^{\infty}(\Omega)}^{2} \leq C$;

(2) $\left\|\nabla \Phi_{i}\right\|_{L^{\infty}(\Omega)}^{2} \leq C / \delta^{2}$;

(3) $\sum_{i=1}^{M} \Phi(x)=1, \forall x \in \bar{\Omega}$;

(4) $\operatorname{supp}\left(\Phi_{i}\right) \subseteq \bar{\Omega}_{i}$.

The following theorem holds (see [11], Lem. 7).

Theorem 3. Let Properties 1 (with $H_{0}=H$ ) and 3 hold. Then, there exists a constant $C>0$ such that

$$
\kappa\left(P_{S, C, \text { add }}^{-1} A\right) \leq C\left(1+\frac{H}{\delta}\right) .
$$

\section{NumericAl EXPERIMENTS}

In this section we report some numerical results for problem (1) with $\Omega=(0,1) \times(0,1)$. The grid is built by dividing $\Omega$ into $n^{2}$ equal squares and subdividing them into two triangles. Thus, we obtain a triangulation with $h=\frac{1}{n}$. As regards the decomposition into subdomains, we consider overlapping squares $\Omega_{i}$ of area $H^{2}$. We use linear finite-elements, and solve the linear system (3) by the conjugate gradient method. Aggregates are build, of square shape, by grouping fine-grid nodes into non-overlapping sets. As a result, the overlap among the aggregates is one element (that is, $\delta_{0}=h$ ).

The tables report the estimated condition number for the preconditioned system; see for instance [7].

We have used minimal overlap among the subdomains, that is, $\delta=h$. Equation (12) is used to define $\tilde{R}_{0}$. The smoother is the Richardson smoother $(14)$, with $\varpi=1.5 / \rho\left(\tilde{A}_{0}\right)$, where 1.5 is a chosen correction factor to minimize the spectral radius of the coarse problem. Possible under-estimate in the value of $\rho(A)$ could be handled by lowering this value.

Table 1 gives the condition number for the one-level Schwarz preconditioner (4). The condition number grows as $\mathcal{O}(1 / h H)$ as theory predicts [16]. The following Tables 2 and 3, which are about the two-level Schwarz preconditioner (5) and (6) with a coarse space built using a coarse grid, confirm the bound provided by Theorem 1. Hybrid preconditioners behave only slightly better than additive ones, therefore their use for these kind of problems seems unjustified. Tables 4 and 5 report the influence of a non-smoothed aggregation procedure. The effect of the parameter $\chi$ of equation (25) can be clearly appreciated. $P_{S, C \text {,hybrid }}^{-1}$ shows the same convergence 
TABLE 1. Estimated condition number for $P_{S}^{-1} A$ using the one-level Schwarz preconditioner $P_{S}$ with minimal overlap $(\delta=h)$.

\begin{tabular}{|c|cccc|}
\hline$P_{S}$ & $H=1 / 2$ & $H=1 / 4$ & $H=1 / 8$ & $H=1 / 16$ \\
\hline$h=1 / 16$ & 15.95 & 27.09 & 52.08 & - \\
$h=1 / 32$ & 31.69 & 54.52 & 104.85 & 207.67 \\
$h=1 / 64$ & 63.98 & 109.22 & 210.07 & 416.09 \\
$h=1 / 128$ & 127.99 & 218.48 & 420.04 & 832.57 \\
\hline
\end{tabular}

TABLE 2. Estimated condition number for $P_{S, C \text {,add }}^{-1} A$, with a coarse space built using a coarse grid (standard coarse space).

\begin{tabular}{|c|cccc|}
\hline$P_{S, C, \text { add }}$ & $H=1 / 4$ & $H=1 / 8$ & $H=1 / 16$ & $H=1 / 32$ \\
\hline$h=1 / 32$ & 7.03 & 4.94 & - & - \\
$h=1 / 64$ & 12.73 & 7.59 & 4.98 & - \\
$h=1 / 128$ & 23.62 & 13.17 & 7.66 & 4.99 \\
$h=1 / 256$ & 45.33 & 24.34 & 13.28 & - \\
\hline
\end{tabular}

TABLE 3. Estimated condition number for $P_{S, C \text {, hybrid }}^{-1} A$, with a coarse built using a coarse grid (standard coarse space).

\begin{tabular}{|c|cccc|}
\hline$P_{S, C, \text { hybrid }}$ & $H=1 / 4$ & $H=1 / 8$ & $H=1 / 16$ & $H=1 / 32$ \\
\hline$h=1 / 32$ & 6.11 & 3.56 & - & - \\
$h=1 / 64$ & 11.47 & 6.24 & 3.58 & - \\
$h=1 / 128$ & 22.26 & 11.71 & 6.27 & 3.58 \\
$h=1 / 256$ & 43.86 & 22.71 & 11.77 & - \\
\hline
\end{tabular}

TABLE 4. Estimated condition number for $P_{S, C \text {, add }}^{-1} A$, with a coarse space built using nonsmoothed aggregation.

\begin{tabular}{|c|c|ccc|}
\hline$P_{S, C, \text { add }}$ & $\chi$ & $H=1 / 4$ & $H=1 / 8$ & $H=1 / 16$ \\
\hline$h=1 / 16$ & 1 & 13.37 & 8.87 & - \\
$h=1 / 32$ & 1 & 26.93 & 17.71 & 9.82 \\
$h=1 / 64$ & 1 & 54.33 & 35.21 & 19.70 \\
$h=1 / 128$ & 1 & 109.39 & 70.22 & 39.07 \\
\hline$h=1 / 32$ & 2 & 13.13 & 7.78 & - \\
$h=1 / 64$ & 2 & 27.18 & 15.28 & 9.96 \\
\hline$h=1 / 32$ & 3 & 7.61 & - & - \\
$h=1 / 64$ & 3 & 17.13 & 8.39 & - \\
\hline
\end{tabular}

rate of the additive version, although the estimated condition number is sensibly smaller. Note that two matrixvector products are needed to apply $P_{S, C \text {, hybrid }}^{-1}$ to a given vector, thus making its application computationally more expensive than that of $P_{S, C \text {,add }}^{-1}$. However, differently from "classical" coarse spaces, hybrid preconditioners perform much better than their additive versions, with significant reduction in the condition number.

Finally, Tables 6-10 show the influence of the smoother (14) for different values of $k$. Note that, for all the smoothers, the hybrid version performs significantly better than the additive one.

By comparing Tables 4 and 6, one can see that one step of smoother (14) results in small reductions in the condition number, using an additive preconditioner. This reduction is even smaller for the hybrid version, 
TABLE 5. Estimated condition number for $P_{S, C \text {,hybrid }}^{-1} A$, with a coarse space built using nonsmoothed aggregation, with $\chi=1$.

\begin{tabular}{|c|ccc|}
\hline$P_{S, C, \text { hybrid }}$ & $H=1 / 4$ & $H=1 / 8$ & $H=1 / 16$ \\
\hline$h=1 / 16$ & 5.24 & 2.89 & - \\
$h=1 / 32$ & 10.64 & 5.66 & 2.97 \\
$h=1 / 64$ & 21.60 & 11.34 & 5.79 \\
$h=1 / 128$ & 43.65 & 22.77 & 11.55 \\
\hline
\end{tabular}

TABLE 6. Estimated condition number for $P_{S, C, \text { add }}^{-1} A$, with a coarse space built using aggregation and smoother (14) with $k=1$ and $\chi=1$.

\begin{tabular}{|c|cccc|}
\hline$P_{S, C, \text { add }}$ & $H=1 / 4$ & $H=1 / 8$ & $H=1 / 16$ & $H=1 / 32$ \\
\hline$h=1 / 16$ & 11.91 & 6.02 & - & - \\
$h=1 / 32$ & 25.59 & 14.95 & 6.28 & - \\
$h=1 / 64$ & 50.03 & 32.64 & 16.23 & 6.36 \\
$h=1 / 128$ & 108.13 & 67.75 & 35.81 & 16.631 \\
$h=1 / 256$ & 218.57 & 137.91 & 74.55 & - \\
\hline
\end{tabular}

TABLE 7. Estimated condition number for $P_{S, C \text {,hybrid }}^{-1} A$, with a coarse space built using aggregation and smoother (14) with $k=1$ and $\chi=1$.

\begin{tabular}{|c|cccc|}
\hline$P_{S, C \text {,hybrid }}$ & $H=1 / 4$ & $H=1 / 8$ & $H=1 / 16$ & $H=1 / 32$ \\
\hline$h=1 / 16$ & 5.09 & 2.86 & - & - \\
$h=1 / 32$ & 10.49 & 5.63 & 2.96 & - \\
$h=1 / 64$ & 21.46 & 11.31 & 5.77 & 2.99 \\
$h=1 / 128$ & 43.51 & 22.75 & 11.54 & 5.82 \\
\hline
\end{tabular}

TABLE 8. Estimated condition number for $P_{S, C \text {,add }}^{-1} A$, with a coarse space built using aggregation and smoother (14) with $k=2$ and $\chi=1$.

\begin{tabular}{|c|cccc|}
\hline$P_{S, C \text {,add }}$ & $H=1 / 4$ & $H=1 / 8$ & $H=1 / 16$ & $H=1 / 32$ \\
\hline$h=1 / 16$ & 10.71 & 5.70 & - & - \\
$h=1 / 32$ & 24.28 & 12.78 & 5.92 & - \\
$h=1 / 64$ & 51.77 & 30.35 & 19.70 & 10.12 \\
$h=1 / 128$ & 106.89 & 65.42 & 32.97 & 13.82 \\
\hline
\end{tabular}

as shown by Tables 5 and 7. This suggests that, at least for the considered problem and two-level hybrid preconditioners, non-smoothed aggregation can be preferred to smoothed aggregation. By comparing Tables 4, 6,8 , and 10 , one can note that high-order polynomials can be used to improve the condition number (even if, for $k>1$, better strategies to define the damping parameters need to be studied).

\section{Conclusions}

In this paper we have presented aggregation procedures to construct the coarse space for two-level Schwarz preconditioners. The basis functions of $V_{0}$ are built as a linear combination of the basis functions of the fine 
TABLE 9. Estimated condition number for $P_{S, C \text {, hybrid }}^{-1} A$, with a coarse space built using aggregation and smoother (14) with $k=2$ and $\chi=1$.

\begin{tabular}{|c|cccc|}
\hline$P_{S, C, \text { hybrid }}$ & $H=1 / 4$ & $H=1 / 8$ & $H=1 / 16$ & $H=1 / 32$ \\
\hline$h=1 / 16$ & 5.00 & 2.82 & - & - \\
$h=1 / 32$ & 10.39 & 5.611 &. & - \\
$h=1 / 64$ & 21.36 & 11.29 & 5.78 & 2.98 \\
\hline
\end{tabular}

TABLE 10. Estimated condition number for $P_{S, C \text {, add }}^{-1} A$, with a coarse space build using aggregation, using smoother (14) with $k=3$ and $\chi=1$.

\begin{tabular}{|c|cccc|}
\hline$P_{S, C, \text { add }}$ & $H=1 / 4$ & $H=1 / 8$ & $H=1 / 16$ & $H=1 / 32$ \\
\hline$h=1 / 16$ & 9.77 & 5.59 & - & - \\
$h=1 / 32$ & 23.12 & 11.08 & 5.88 & - \\
$h=1 / 64$ & 50.56 & 28.31 & 11.55 & - \\
\hline
\end{tabular}

space, and the use of a coarse triangulation is not required. For this reason, aggregation procedures can be easily applied to problems defined on complex geometries without losing the power of two-level methods. The coarse matrix can be constructed automatically and for any computational grid with no input from the user, except for the linear system matrix $A$ and the dimension of the coarse space. Moreover, the computational complexity of the aggregation procedure is smaller since the method is simpler to implement. This simplicity has its origin in the way the restriction and interpolation operators are defined.

A theoretical analysis is reported for an elliptic model problem, discretized on a quasi-uniform grid with finite-elements. Our theory extends results presented in literature; see [8,11]. There, the authors assume equal overlap among the subdomains $\delta$ and the aggregates $\delta_{0}$, and equal size of the subdomains $H$ and the aggregates $H_{0}$. Instead, in the bound of Theorem 2, the influence of the subdomain decomposition and the aggregates are kept separate.

For the special case $H=H_{0}$ and $\delta=\delta_{0}$, Theorem 3 furnishes an improved convergence bound, which depends linearly on $H / \delta$. However, the basis functions of the coarse space must satisfy different properties: Property 2 for Theorem 2 and Property 3 for Theorem 3. It can be proved that the presented smoother satisfies Property 2 , while, to our knowledge, there are no proofs that it satisfies Property 3. (We note however that this property can be verified for special cases.) Theorem 2 is more general and allows aggregates and subdomains of different shapes and overlap, but numerical results (at least for our model problem) seem to satisfy Theorem 3.

Acknowledgements. The author wants to thank the anonymous referees for their helpful suggestions, that helped to improve the quality of this paper.

\section{REFERENCES}

[1] M. Brezina, Robust iterative method on unstructured meshes. Ph.D. Thesis, University of Colorado at Denver (1997).

[2] M. Brezina and P. Vaněk, A black-box iterative solver based on a two-level Schwarz method. Computing 63 (1999) $233-263$.

[3] O. Broeker, M.J. Grote, C. Mayer and A. Reusken, Robust parallel smoothing for multigrid via sparse approximate inverses. SIAM J. Sci. Comput. 23 (2001) 1396-1417.

[4] M. Dryja and O.B. Widlund, Domain decomposition algorithms with small overlap. SIAM J. Sci. Comput. 15 (1994) 604-620.

[5] M. Dryja and O.B. Widlund, Schwarz methods of Neumann-Neumann type for three-dimensional elliptic finite element problems. Comm. Pure Appl. Math. 48 (1995) 121-155.

[6] L. Formaggia, A. Scheinine and A. Quarteroni, A numerical investigation of Schwarz domain decomposition techniques for elliptic problems on unstructured grids. Math. Comput. Simulations 44 (1994) 313-330.

[7] G.H. Golub and C.F. van Loan, Matrix Computations. The Johns Hopkins University Press, Baltimore, Maryland (1983). 
[8] L. Jenkins, T. Kelley, C.T. Miller and C.E. Kees, An aggregation-based domain decomposition preconditioner for groundwater flow. Technical Report TR00-13, Department of Mathematics, North Carolina State University (2000).

[9] C.E. Kees, C.T. Miller, E.W. Jenkins and C.T. Kelley, Versatile multilevel Schwarz preconditioners for multiphase flow. Technical Report CRSC-TR01-32, Center for Research in Scientific Computation, North Carolina State University (2001).

[10] C. Lasser and A. Toselli, An overlapping domain decomposition preconditioner for a class of discontinuous Galerkin approximations of advection-diffusion problems. Technical Report 810, Dept. of Computer Science, Courant Institute (2000). Math. Comput. 72 (2003) 1215-1238.

[11] C. Lasser and A. Toselli, Convergence of some two-level overlapping domain decomposition preconditioners with smoothed aggregation coarse spaces. Technical Report TUM-M0109, Technische Universität München (2001).

[12] W. Leontief, The structure of the American Economy. Oxford University Press, New York (1951).

[13] J. Mandel and B. Sekerka, A local convergence proof for the iterative aggregation method. Linear Algebra Appl. 51 (1983) $163-172$.

[14] L. Paglieri, A. Scheinine, L. Formaggia and A. Quarteroni, Parallel conjugate gradient with Schwarz preconditioner applied to fluid dynamics problems, in Parallel Computational Fluid Dynamics, Algorithms and Results using Advanced Computer, Proceedings of Parallel CFD'96, P. Schiano et al., Eds. (1997) 21-30.

[15] A. Quarteroni and A. Valli, Numerical Approximation of Partial Differential Equations. Springer-Verlag, Berlin (1994).

[16] A. Quarteroni and A. Valli, Domain Decomposition Methods for Partial Differential Equations. Oxford University Press, Oxford (1999).

[17] M. Sala and L. Formaggia, Parallel Schur and Schwarz based preconditioners and agglomeration coarse corrections for CFD problems. Technical Report 15, DMA-EPFL (2001).

[18] M. Sala and L. Formaggia, Algebraic coarse grid operators for domain decomposition based preconditioners, in Parallel Computational Fluid Dynamics - Practice and Theory, P. Wilders, A. Ecer, J. Periaux, N. Satofuka and P. Fox, Eds., Elsevier Science, The Netherlands (2002) 119-126.

[19] B.F. Smith, P. Bjorstad and W.D. Gropp, Parallel Multilevel Methods for Elliptic Partial Differential Equations. Cambridge University Press, Cambrige (1996).

[20] P. Le Tallec, Domain decomposition methods in computational mechanics, in Computational Mechanics Advances, J.T. Oden, Ed., North-Holland 1 (1994) 121-220.

[21] R.S. Tuminaro and C. Tong, Parallel smoothed aggregation multigrid: Aggregation strategies on massively parallel machines, in SuperComputing 2000 Proceedings, J. Donnelley, Ed. (2000).

[22] P. Vanek, M. Brezina and R. Tezaur, Two-grid method for linear elasticity on unstructured meshes. SIAM J. Sci. Comput. 21 (1999) 900-923.

[23] P. Vanek, M. Brezina and J. Mandel, Convergence of algebraic multigrid based on smoothed aggregation. Numer. Math. 88 (2001) 559-579.

To access this journal online:

www.edpsciences.org 